\title{
Heat transfer by unsteady laminar mixed convection in $2-D$ ventilated enclosures using the vorticity-stream function formulation
}

\author{
S. Boudebous \& Z. Nemouchi \\ Département de Génie Mécanique, \\ Université Mentouri de Constantine Algéria, Algeria
}

\begin{abstract}
In this work, a numerical study is presented of mixed laminar convection in ventilated enclosures. The left vertical wall of enclosure is maintained at a constant temperature greater than that of the fluid at entry, while the other walls are adiabatic. Two cases of ventilation are considered. In the first case, the fluid enters from the bottom left corner and leaves the domain through the right upper corner. In the second case, the fluid enters from the bottom left corner and leaves the domain through the middle of the upper wall.

The equations governing the phenomenon are discretised using the finitedifference method. A computer programme is developed to simulate the flow behaviour and the heat transfer in the enclosure. Velocity and temperature fields are obtained. These numerical simulations are performed for a Grashof number of $10^{6}$, a Prandlt number of 0.7 and three values of the Richardson number $(0.5$, 5 , and 25)

Keywords: mixed convection, vorticity-stream function formulation, square enclosure, numerical method.
\end{abstract}

\section{Introduction}

An interesting review on natural convection in enclosures can be found in Fusegi and Hyun [1]. Concerning the subject of the present work, numerous investigations have been reported. We can cite as examples:

Unsteady 2-D hot water flow for energy extraction from a storage system, Cha and Jaluria [2] with cold water inlet at the bottom of the left vertical wall and hot water exit at the top of the same or opposite wall. Unsteady 2-D flow of air, Raji 
and Hasnaoui [3] with inlet at the top of the left vertical wall and exit at the top or bottom of the opposite wall. Transient mixed convection, Omri and Ben Nasrallah [4] with air inlet at the top (at the bottom in a second case) of the hot left wall and exit at the bottom (at the top in the second case) of the right cold wall. Air flow with openings at the top of the vertical walls with one or two heat sources of zero thickness imbedded on a vertical board of finite thickness placed on the bottom wall, Hsu and Wang [5]. In the present study, laminar mixed convection flow of air in a ventilated cavity has been investigated numerically. The physical model under consideration and coordinates chosen are depicted in figure 1 .
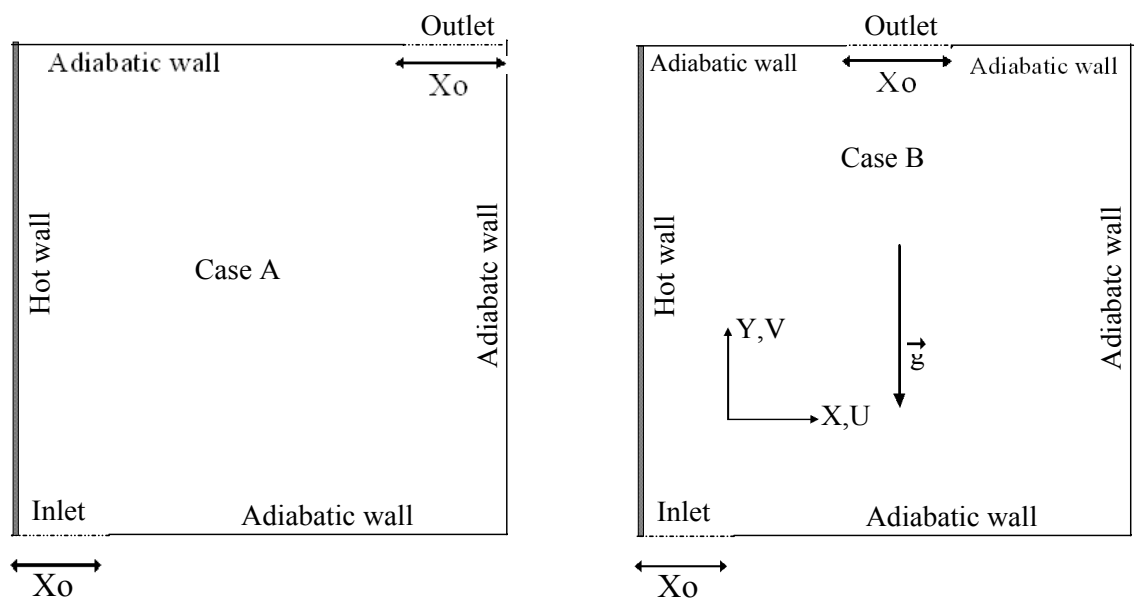

Figure 1: Geometrical configuration.

\section{Governing equations}

The flow and heat transfer phenomena to be investigated here are described by the complete Navier-Stokes and energy equations for two-dimensional laminar incompressible flows. The viscous dissipation term in the energy equation is neglected and the Boussinesq approximation is invoked for the buoyancy induced body force term in the Navier-Stokes equations.

From the governing equations of mass, momentum conservations, the vorticity-stream function formulation may be obtained by defining the stream function and vorticity, as, respectively,

$$
U=\frac{\partial \psi}{\partial Y} \quad V=-\frac{\partial \psi}{\partial X} \quad \omega=\frac{\partial V}{\partial X}-\frac{\partial U}{\partial Y}
$$

Hence, the equations in dimensionless form can be written as follows.

Stream function equation

$$
\frac{\partial^{2} \psi}{\partial X^{2}}+\frac{\partial^{2} \psi}{\partial Y^{2}}=-\omega
$$


Vorticity-transport equation

$$
\frac{\partial \omega}{\partial \tau}+U \frac{\partial \omega}{\partial X}+V \frac{\partial \omega}{\partial Y}=\frac{1}{\operatorname{Re}}\left(\frac{\partial^{2} \omega}{\partial X^{2}}+\frac{\partial^{2} \omega}{\partial Y^{2}}\right)+R i \frac{\partial \theta}{\partial X}
$$

Energy equation

$$
\frac{\partial \theta}{\partial \tau}+U \frac{\partial \theta}{\partial X}+V \frac{\partial \theta}{\partial Y}=\frac{1}{\operatorname{Re} \operatorname{Pr}}\left(\frac{\partial^{2} \theta}{\partial X^{2}}+\frac{\partial^{2} \theta}{\partial Y^{2}}\right)
$$

where Re, Ri and Pr denote, respectively, Reynolds number, Richardson number and Prandlt number. They are defined as

$$
\operatorname{Re}=\frac{V_{o} L}{v} \quad \operatorname{Pr}=\frac{v}{\alpha} \quad R i=\frac{G r}{\operatorname{Re}^{2}} \quad G r=\frac{g \beta\left(T_{w}-T_{o}\right) L^{3}}{v^{2}}
$$

Here Gr is the Grashof number.

The other dimensionless parameters are defined as follows:

$$
X=\frac{x}{L} \quad Y=\frac{y}{L} \quad U=\frac{u}{V_{o}} \quad V=\frac{v}{V_{o}} \quad \theta=\frac{T-T_{o}}{T_{w}-T_{o}} \quad \tau=\frac{V_{o} t}{L}
$$

where $\mathrm{L}$ is the characteristic length of the cavity, $\mathrm{V}_{\mathrm{o}}$ is the inlet velocity, $v$ is the kinematic viscosity, $\alpha$ is the thermal diffusivity, $g$ is the acceleration of gravity, $\beta$ is the thermal expansion coefficient, $T_{w}$ is the wall temperature, $T_{o}$ is the temperature of the fluid at the entry and $t$ is the time.

For the problem geometry, the following boundary conditions are specified:

$$
\begin{gathered}
U=0 . \quad V=0 . \quad \theta=1 . \quad \Psi=0 . \quad \text { at } \quad X=0 . \quad 0 \prec Y \prec 1 . \\
U=0 . \quad V=0 . \quad \frac{\partial \theta}{\partial X}=0 . \quad \Psi=\Psi_{x o} \quad \text { at } \quad X=1 . \quad 0 \prec Y \prec 1 . \\
U=0 . \quad V=1 . \quad \theta=0 . \quad \Psi=-X \quad \omega=0 . \quad \text { at } \quad Y=0 . \quad 0 \prec X \prec X_{O}
\end{gathered}
$$




$$
\begin{gathered}
U=0 . \quad V=0 . \quad \frac{\partial \theta}{\partial Y}=0 . \quad \Psi=\Psi_{x o} \quad \text { at } \quad Y=0 . \quad X_{O} \prec X \prec 1 . \\
U=0 . \quad V=0 . \quad \frac{\partial \theta}{\partial Y}=0 . \quad \Psi=0 . \quad \text { at } \quad Y=1 . \quad 0 \prec X \prec 1 .-X_{O} \\
\frac{\partial^{2} \Phi}{\partial Y^{2}}=0 . \quad \text { at } \quad Y=1 . \quad 1 .-X_{O} \prec X \prec 1 .
\end{gathered}
$$

where $\Phi$ stands for $\theta, \omega, \psi, U$ and $V$. The width of the inlet and the outlet Xo is equal to 0.1 .

In general, the value of vorticity on a solid boundary is deduced from Taylor series expansion of the stream function around the solid point and can be expressed mathematically as

$$
\omega_{\text {wall }}=-\frac{\partial^{2} \psi}{\partial n^{2}}
$$

where $\mathrm{n}$ is the outward drawn normal of the surface.

The convective heat transfer from the heated wall can be characterized by an average Nusselt number, Num, defined as

$$
N u m=\int_{0}^{1}\left[-\frac{\partial \theta}{\partial X}\right]_{X=0} d Y
$$

\section{Numerical procedure}

The governing equations (2-4) along with the boundary conditions (7-13) are solved numerically, employing finite-difference techniques. The Alternating Direction Implicit (A.D.I.) method of Peaceman and Rachford in [6] is used for time marching. The buoyancy and diffusive terms are discretized by using central differencing while the use of a third-order upwind scheme [7] is preferred for convective terms. Convergence of iteration for the stream function solution is obtained at each time step. The resulting set of finite difference equations is then solved by using the Non Linear Over Relaxation (N.L.O.R.) method [8].

All computations are performed using non-uniform grids with denser clustering near the walls where boundary layers develop and high gradients are expected [9].

The solutions were initially tested with mesh sizes of $81 \times 81,101 \times 101$, $121 \times 121$ and $151 \times 151$. It was found that variations in the solution fields were not significant (of the order of $1 \%$ in the mean Nusselt number obtained) between 
mesh sizes of $121 \times 121$ and $151 \times 151$. As a compromise between accuracy and CPU time, the mesh size of $121 \times 121$ is used for all calculations.

The validity of the computer code developed has been checked for the sudden expansion of an oblique velocity field in a cavity [10] and the buoyancy-driven cavity flow [11]. The various sets of results compare very well and are nearly identical, confirming the credibility of the code.

\section{Numerical results and discussion}

The main characteristics of the flow and energy transport for each Richardson number (Ri) and for each case of ventilation will be shown in the following. The mean Nusselt numbers (Num) are shown as plots versus the time (figure 2). Flow and temperature fields are shown in terms of stream traces, isotherms, velocity and temperature profiles (figures 3-6).

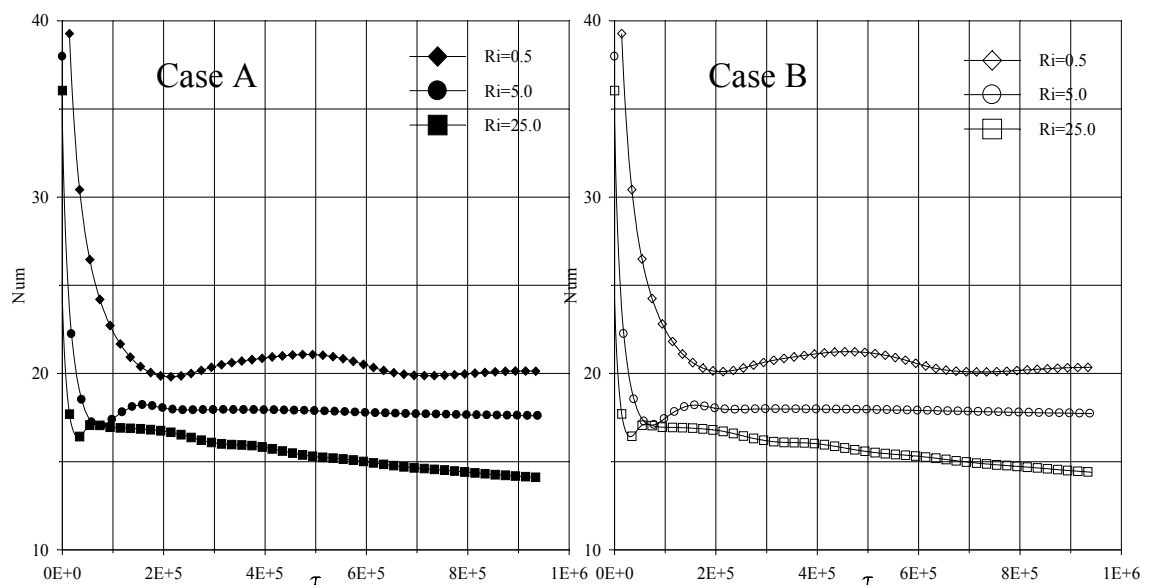

Figure 2: $\quad$ Mean Nusselt numbers versus time.

In both cases $\mathrm{A}$ and $\mathrm{B}$, figure 2 shows that as Ri increases, Num decreases implying that the more the forced convection is dominant, the more important is the heat flux from the hot wall.

Figure 3 shows the stream traces and the profiles of the velocity components $\mathrm{U}$ and $\mathrm{V}$ in the $\mathrm{X}$ and $\mathrm{Y}$ directions respectively. As Ri increases, a boundary layer forms along the hot vertical wall. The relatively fast circulation in the center of the enclosure for $\mathrm{Ri}=0.5$ becomes slower for $\mathrm{Ri}=5$ and 25 . Consistently with this result, the isotherms and the temperature profiles (figure 4) show that an essentially conductive heat transfer for $\mathrm{Ri}=25$ takes over a forced convection for $\mathrm{Ri}=0.5$. It is clear that in case B the effect of Ri on the flow and the heat transfer is very similar to that in case A (figures 5 and 6) 
38 Advanced Computational Methods in Heat Transfer IX
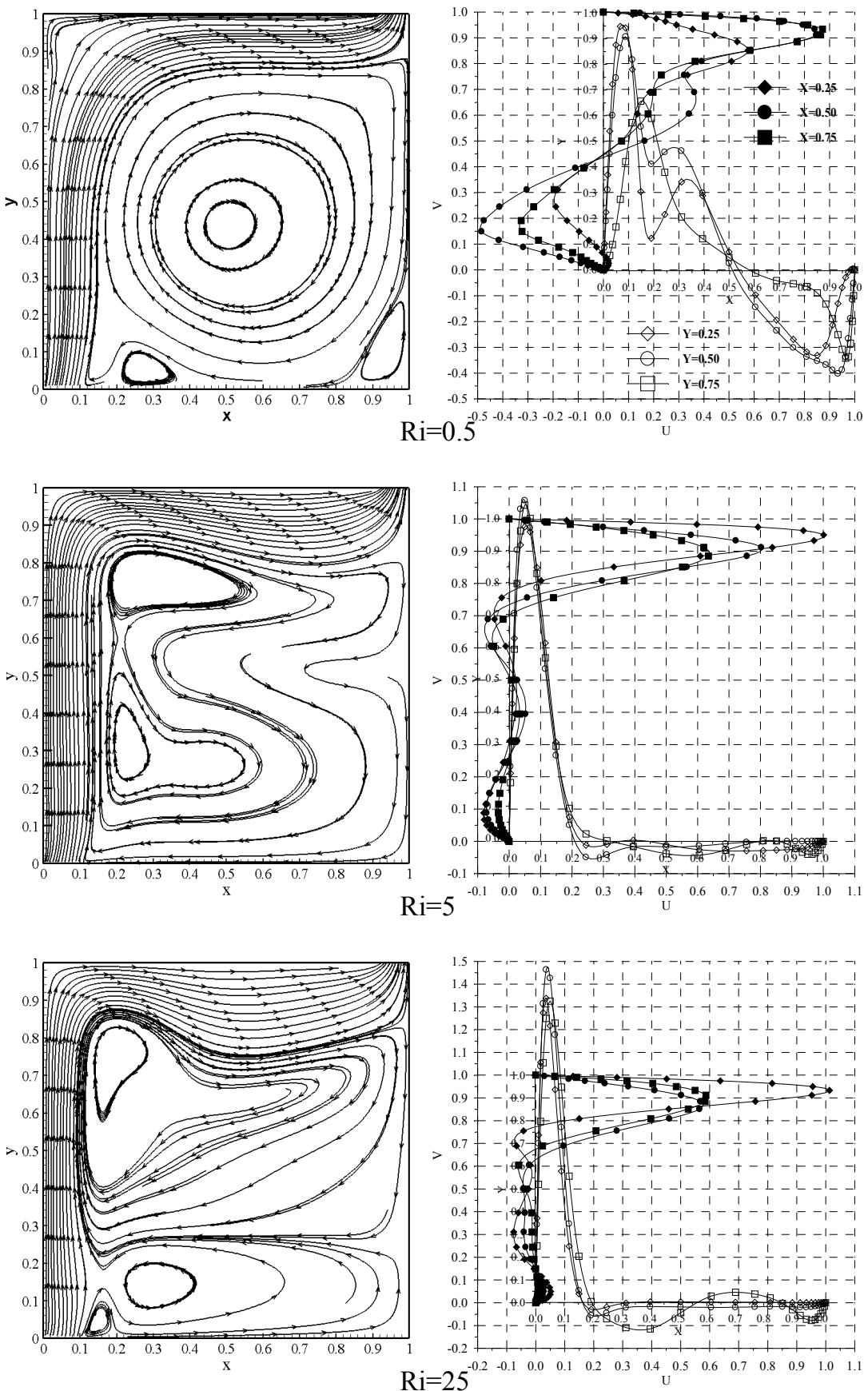

Figure 3: $\quad$ Stream traces (left) and Velocity profiles (right) case A. 

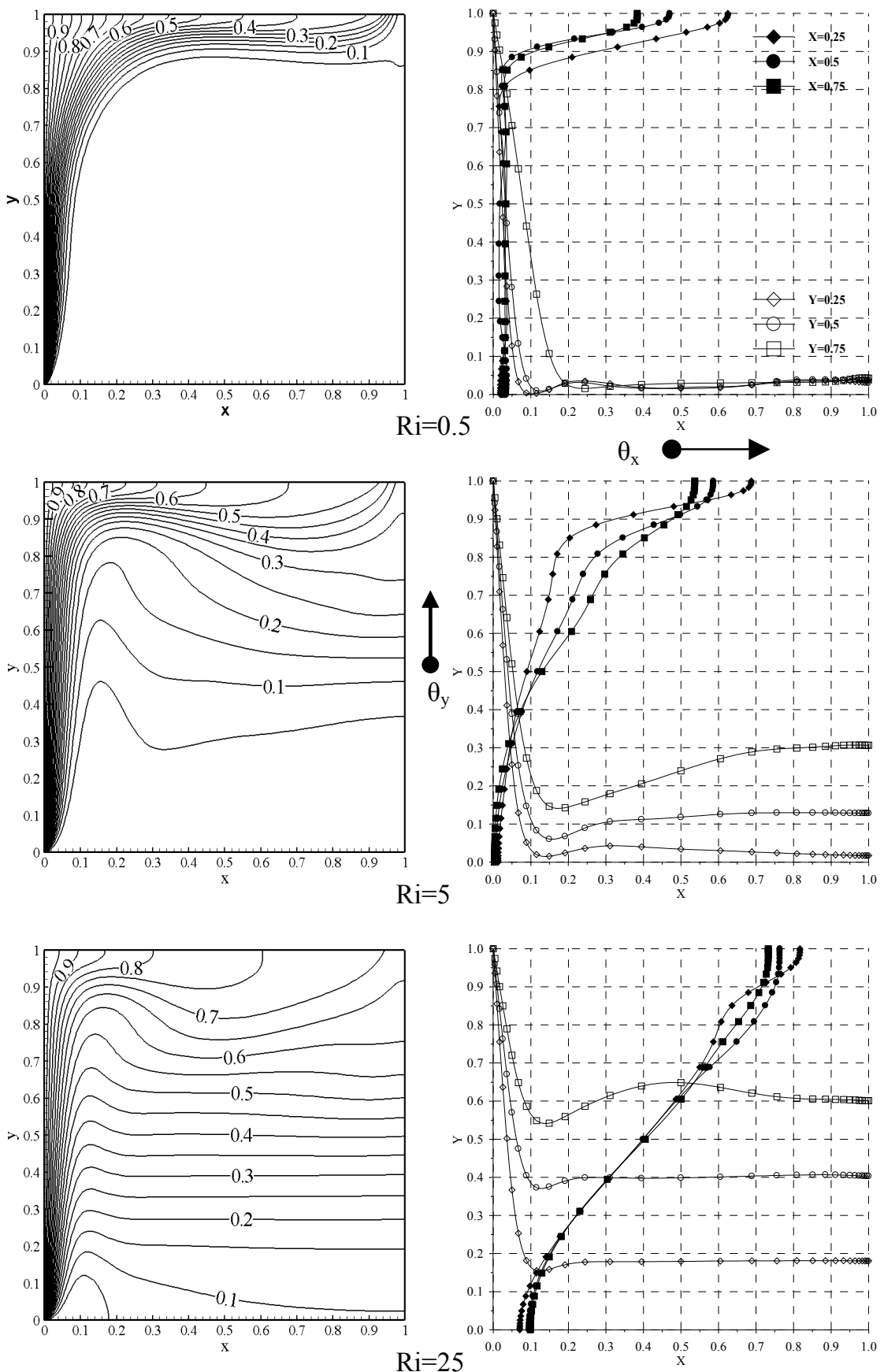

Figure 4: $\quad$ Isotherms (left) and Temperature profiles (right) case A. 

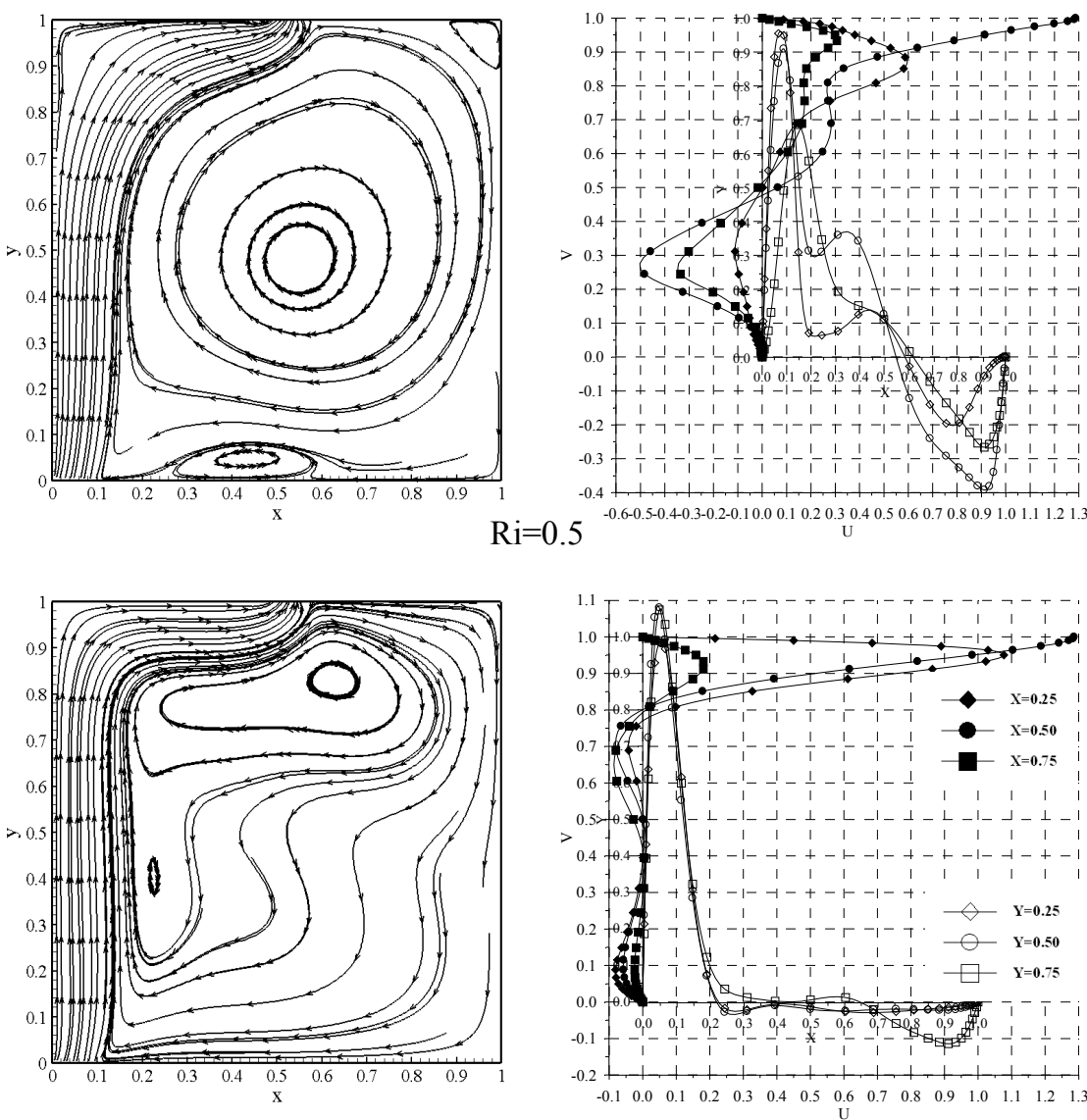

$\mathrm{Ri}=5$
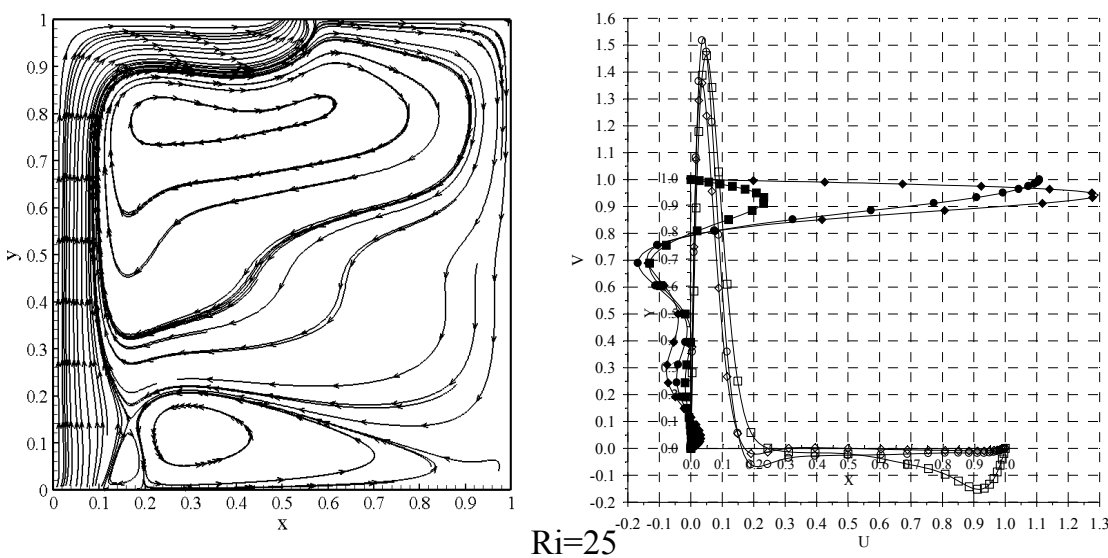

Figure 5: $\quad$ Stream traces (left) and Velocity profiles (right) case B.

WIT Transactions on Engineering Sciences, Vol 53, (C) 2006 WIT Press www.witpress.com, ISSN 1743-3533 (on-line) 

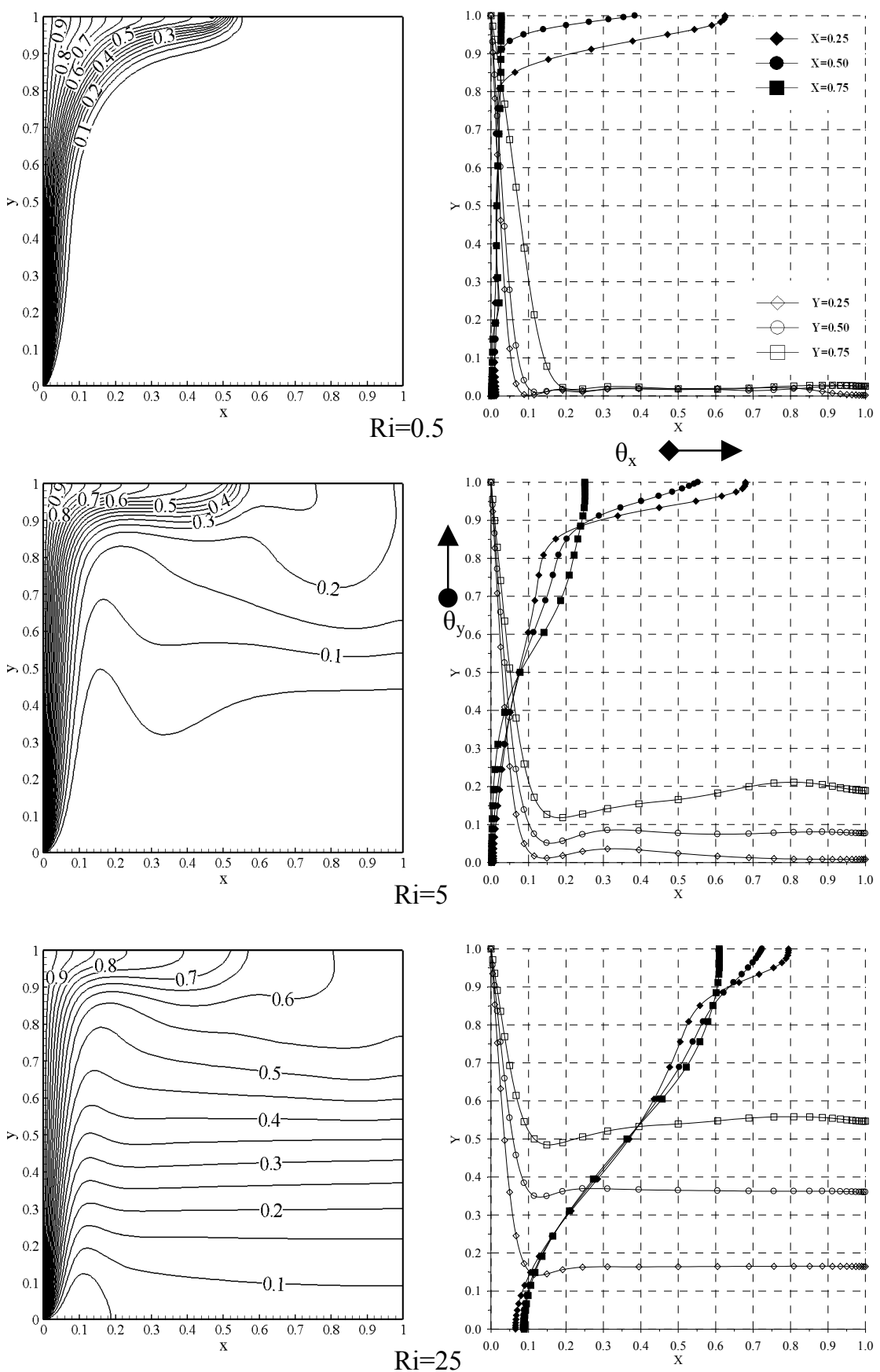

Figure 6: Isotherms (left) and Temperature profiles (right) case B. 


\section{References}

[1] Fusegi, T. \& Hyun, J.M., Laminar and transitional natural convection in an enclosure with complex and realistic conditions, Int. J. Heat Fluid Flow. 3, pp. 258-268, 1994

[2] Cha, C. K. \& Jaluria, Y., Recirculating mixed convection flow for energy extraction. Int. J. Heat Mass Transfer. 27, pp. 1801-1812, 1984

[3] Raji, A. \& Hasnaoui, M., Correlations en convection mixte dans des cavités ventilées. Rev. Gen. Therm. 37, pp. 874-884, 1998

[4] Omri, A. \& Ben Nasrallah, S., Control volume finite element numerical simulation of mixed convection in an air-cooled cavity. Numerical Heat Transfer, Part A, vol.36, pp. 615-637, 1999.

[5] Hsu, T. H. \& Wang, S. G., Mixed convection in a rectangular enclosure with discrete heat sources. Numerical Heat Transfer, Part A, vol.38, pp. 627-652, 2000.

[6] Peaceman, D.W. \& Rachford, H.H., Numerical solution of parabolic and elliptic differential equations, J. Soc. Indust. Appl. Math. 3, pp. 28-41, 1955.

[7] Kawamura, T. \& Kawamura, K., Computations of high Reynolds number flow around a circular cylinder with surface roughness, AIAA Paper No 84-0340, 1984.

[8] Sewell, G., The numerical solution of ordinary and partial differential equations, Academic Press, INC, New-York, 1988.

[9] Manole, D.M. \& Lage, L.L., Nonuniform grid accuracy test applied to the natural convection flow within a porous medium cavity, Numerical Heat Transfer, Part B,vol.23,pp. 351-368, 1993.

[10] Song, B., Liu, G.R. \& Lam, K.Y., Four-point interpolation schemes for convective fluxes, Numerical Heat Transfer, Part B,vol.35,pp. 23-39, 1999.

[11] Rahman, M.M., Miettinen, A. \& Siikonen, T., Modified SIMPLE formulation on a collocated grid with an assessment of the simplified Quick scheme, Numerical Heat Transfer, Part B,vol.30,pp. 291-314, 1996. 